\title{
Design of Motor Condition Online Monitoring System Based on LabVIEW
}

\author{
Fengyun XIE ${ }^{1}$, Shaoshi YAN, Jiankun DONG, Yongqi JIANG, and Yu FU \\ School of Mechatronics and Vehicle Engineering, East China Jiaotong University, \\ Jiangxi, Nanchang 330013, China
}

\begin{abstract}
In the rail transit industry, the electric locomotive's electric motor is used as the driving core, and its installation and working environment are often harsh, and the probability of failure will increase accordingly. If the motor fails during the operation of the locomotive, it will cause huge economic losses, and even threaten people's lives and property. In order to grasp the running status of the motor in real time, an online monitoring system of the motor status is designed. This system collects the three parameters of temperature, current and speed, and inputs the three-parameter data into the single-chip microcomputer. The single-chip microcomputer transmits the data to the host computer through calculation. The host computer analyzes and displays the data through LabVIEW to realize online monitoring of the motor status. Experiments show that the system has a good man-machine interface, a wide speed range, high accuracy, fast response speed, and strong anti-interference ability, which is of great practical value for online monitoring of motors.
\end{abstract}

Keywords: Motor, microcontroller, LabVIEW, online monitoring

\section{Introduction}

As a kind of electrical equipment that can convert electrical energy into mechanical energy, the motor plays a great role in industrial production [1]. Once the motor fails during operation, industrial production is difficult to carry out safely, so it is necessary to design a remote motor condition monitoring system [2]. With the improvement of computer level, the technology of combining virtual instruments and sensors has been widely used in the field of equipment monitoring [3]. Because of its graphical programming, LabVIEW is more flexible and convenient than VC, VB and other programming languages, so it has great advantages in multi-sensor acquisition and data analysis [4].

The lower computer of the system obtains data of three parameters of temperature, current and speed during the operation of the motor through the sensor and the conditioning circuit. Then it processes the data through the single-chip computer and transmits the data to the upper computer using the wireless transmission module. And the upper computer monitoring software select LabVIEW to display the motor temperature, current and speed parameters.

1 Fengyun Xie, School of Mechatronics and Vehicle Engineering, East China Jiaotong University, Jiangxi, Nanchang 330013, China. Email: 1040449239@qq.com 


\section{System Overall Structure Design}

The motor status remote monitoring system mainly includes the following four parts: data acquisition module, data acquisition and processing module, wireless transmission module and host computer LabVIEW interface.

Among them, the data acquisition module mainly obtains the temperature parameters through the Pt100 platinum thermal resistance temperature sensor [5], the current parameters through the TA12-100 current transformer, and the speed parameters through the Hall speed sensor [6], through the signal The conditioning is converted into signals that can be collected by the microcontroller.

The data acquisition module mainly uses the STM32F103 model single-chip microcomputer for data acquisition and processing functions [7].

The wireless transmission module mainly realizes its wireless transmission function through the NRF24L01 radio frequency module. This module mainly transmits the data processed by the sending end Microcontroller Unit (MCU) to the receiving end MCU through wireless transmission.

LabVIEW software is used in the upper computer. The function of LabVIEW software is to receive the data sent by the single-chip microcomputer at the receiving end through wired transmission and to process the threshold value, and then display the data and results on the front panel. After the motor starts to work, the three kinds of sensors acquire the parameters of temperature, speed and current respectively, and then convert them into analog signals and input them to STM32 single-chip microcomputer through the conditioning circuit. The single-chip microcomputer restores the signals to the corresponding physical quantity digital quantity, and then transmits the data to the computer through the wireless transmission module. The computer displays the running state of the motor through the LabVIEW visual interface and parameters. The overall structure diagram of the system is shown in Figure 1.

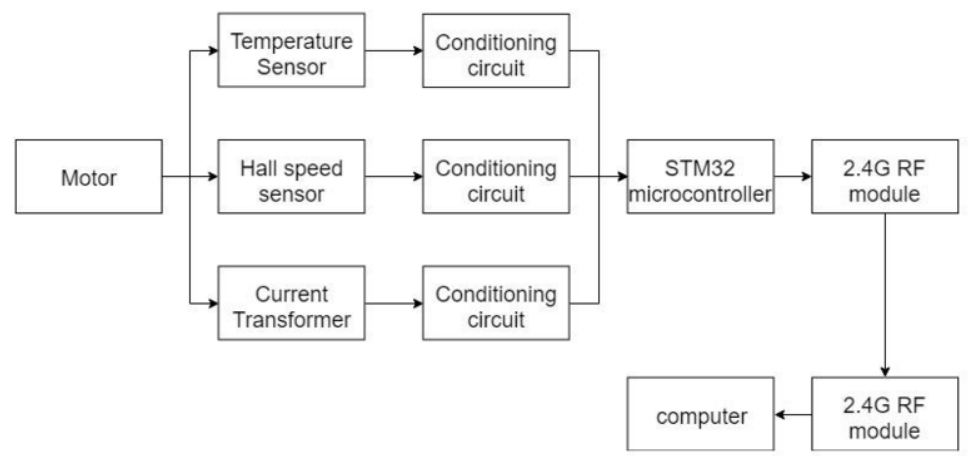

Figure 1.Overall structure of the system

\section{Design of Hardware Circuit of the System}

\subsection{Design Ideas of the Hardware Circuit}

The hardware circuit design of the system mainly involves PT100 platinum thermistor temperature sensor, ta12-100 current transformer, Hall speed sensor, STM32 single 
chip microcomputer and RF module.

This design selects the Pt100 platinum thermal resistance temperature sensor to obtain the temperature parameters during the operation of the motor in real time, converts the temperature change into a voltage value change through the bridge circuit, and then outputs the analog signal with a more obvious change through the differential amplifier circuit and converts it Input to the PB0 port $[8,9]$ of the sending microcontroller, used for A / D conversion; select TA12-100 current transformer to obtain the current parameters in real time during the operation of the motor, and build a zero-crossing with LM311 as the core The comparison circuit adjusts the output alternating voltage to eliminate the negative half-cycle voltage, and inputs the signal with only the positive half-cycle alternating voltage value after the conditioning to the PB1 port of the sending end microcontroller; the Hall speed sensor is selected for the operation of the motor. The speed parameter is acquired in real time, and the obtained speed pulse signal is input to the PA0 port of the sending microcontroller. After the MCU at the sending end collects the analog voltage value signal output by the conditioning circuit, the analog voltage value and the pulse signal are converted into digital and pulse frequency values through the A / D conversion chip and its peripheral circuit and input capture function, respectively, and the digital The quantity and pulse frequency value are wirelessly sent to the receiving MCU through the $2.4 \mathrm{G}$ NRF24L01 radio frequency module.

After the receiving MCU receives the data output by the sending end, the data is spliced to obtain complete numerical data, and then the numerical data is restored to three parameters of temperature, current and rotational speed according to the temperature formula, current formula and speed pulse relationship, and finally the three parameters are sent to the computer for display by wire. System hardware circuit design diagram shown in Figure 2.

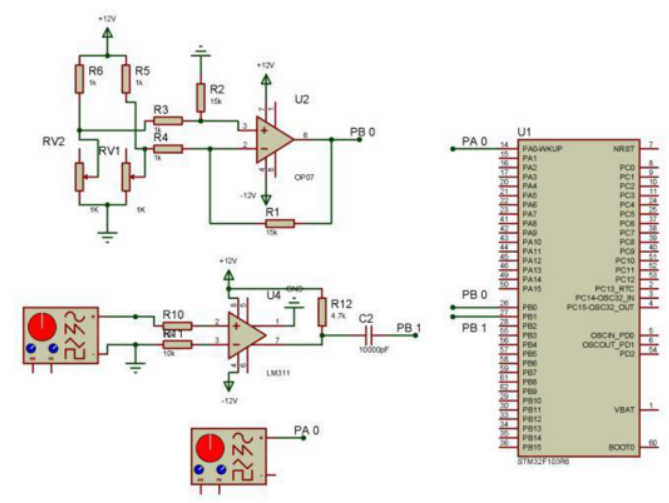

Figure 2. System hardware circuit design

\subsection{Introduction of System Hardware}

This section will introduce the various hardware used in this system:

Introduction of the core module: The STM32 microcontroller is an excellent processor with strong stability, low price and high-speed processing capability. This single-chip microcomputer integrates a 256-byte flash memory. Compared with other series of single-chip microcomputers, the data processing and transmission speed have 
been greatly improved. It has four general-purpose timers, two high-speed timers, three 12-bit precisions, two DMA channels and $64 \mathrm{I} / \mathrm{O}$ ports.

The STM32 microcontroller system supports multiple triggering methods and can use the channels of the ADC1 and ADC2 AD conversion chips at the same time to improve the efficiency of data processing and conversion, and can be called separately The DMA channel reduces the CPU usage rate of the single-chip microcomputer and improves the efficiency of data transfer between registers. The upper limit of the clock frequency of the $\mathrm{AD}$ conversion chip embedded in the single-chip microcomputer is $14 \mathrm{MHz}$, and it has a variety of sampling time settings.

Introduction of the temperature monitoring module: The temperature measurement module of this design uses a Pt100 platinum thermal resistance temperature sensor. This sensor has a monitoring accuracy of $\pm 0.15^{\circ} \mathrm{C}$, stable performance, and the temperature range that can be felt is between -20 and $450^{\circ} \mathrm{C}$, with small errors. Compared with other sensors, the Pt100 sensor has high accuracy and simple wiring. Even in a harsh environment, it can still achieve its temperature measurement function stably. However, it is necessary to build a resistance-voltage conversion circuit when using it, and to control the output voltage value to zoom in. In order to compensate for temperature drift and suppress zero drift during use, when building the hardware circuit, use a bridge circuit for resistance-voltage conversion. In order to obtain more obvious numerical changes, you should also add a differential amplifier circuit.

Introduction of the current monitoring module: The current monitoring module of this design uses TA12-100 current transformer, which is based on the Hall principle to obtain current data, and has the advantages of good stability, accurate measurement, and non-contact measurement. The TA12-100 current transformer uses the VCC port of the single-chip microcomputer for power supply. It needs to form a common ground loop with the single-chip microcomputer during using, otherwise there is no output that can be monitored.

Introduction of the speed monitoring module: The speed measurement module of this design uses the Hall speed measurement sensor, which has a wide monitoring range, high sensitivity and fast response speed. In the design, the magnet is installed on the elongated shaft of the motor, and the Hall speed sensor is used to align the magnet vertically to detect whether there is a magnet sweeping the sensing area. If the magnet is detected, a pulse is output. The Hall speed sensor is small in size, accurate in detection, and stable in performance. It can detect higher speeds and is not affected by dust and oil.

Introduction of the wireless transmission module: The NRF24L01 radio frequency module uses serial communication to read data from the microcontroller to the sending buffer, and after receiving the data, puts the data in the receiving buffer for the microcontroller to read using the serial port. The chip has a small size and a lower price than WiFi modules and other remote communication modules. The transmission distance can reach up to 500 meters, and it can reach 1,800 meters under extreme conditions, and the transmission speed is fast.

\section{LabVIEW Program Design of Upper Computer}

Because LabVIEW integrates the bus protocols such as VXI, GPIB and USB and all the functions of the data acquisition card, it also has built-in library functions such as RS232 serial port and Active $\mathrm{X}$ that are easy to apply. Therefore, this system uses 
LabVIEW as the monitoring interface, which not only greatly saves the user's development time, but also has a friendly interface and simple operation[10]. The LabVIEW program designed by this system is mainly composed of serial port receiving module program, global variable module program, data saving module design and threshold comparison module program.

\subsection{Serial Port Receiving Module Program}

After setting the input control and baud rate selection input control in the serial port receiving module, the upper computer can receive the data transmitted by the single chip microcomputer by running the serial port receiving program.

In order to put the received data of three parameters into the corresponding sensor data buffer, the string index must be carried out by the sensor identification bit of the received data. The serial port configuration is shown in Figure 3. Data bit extraction is shown in Figure 4.

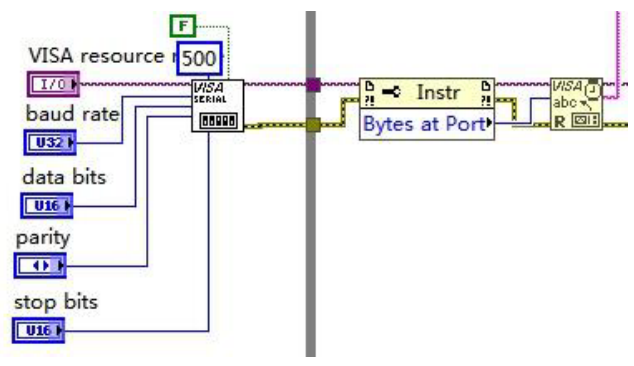

Figure 3. Serial port configuration

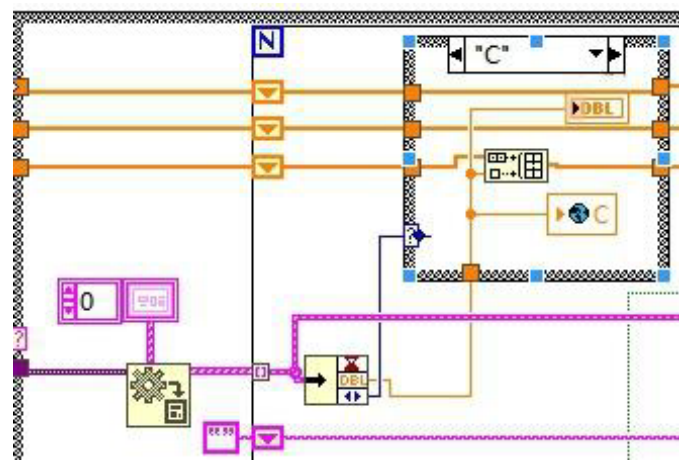

Figure 4.Data bit extraction

\subsection{Global Variable Module Program}

The global variable is set so that the acquired data can be calculated and compared in the main program. First, the temperature, current and speed data obtained by the upper computer are written into the global variables, and then the data is called out from the global variables for threshold comparison when it is needed. The global variable writer in the serial port receiving module program is shown in Figure 5, and the global variable calling program is shown in Figure 6. 


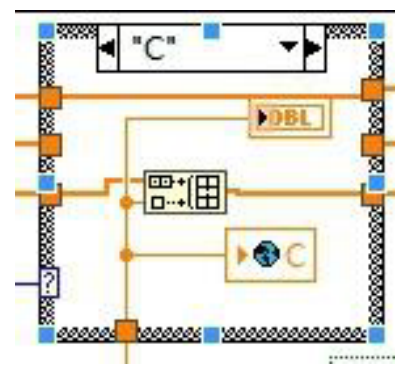

Figure 5. Global variable writing program

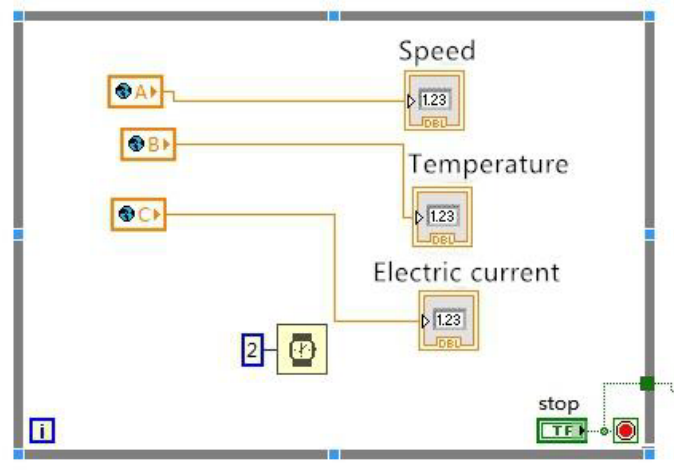

Figure 6. Global variable calling program

\subsection{Data Saving Module Design}

When the main VI receives the data sent by the sub VI from the serial port and performs threshold analysis and display, the user can save the received data to the corresponding path through the data saving module. The program diagram of data saving module is shown in Figure 7.

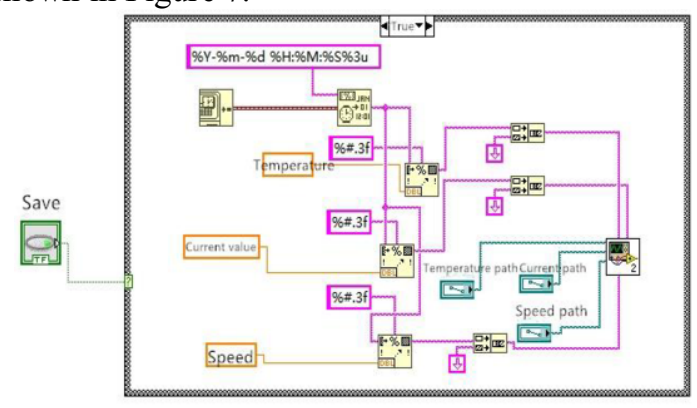

Figure 7. Data saving module design

\subsection{Threshold Comparison Module Program}

In the threshold comparison module, a lower threshold and an upper threshold will be set for temperature, current and speed. When the serial port receiving program receives the three parameters transmitted by the single chip microcomputer, it will store the effective data bits in the global variables, and then the threshold comparison module 
calls the data in the global variables to compare with the previously preset threshold value. When the value is lower than the lower threshold value or higher than the upper threshold value, the fault warning light of the corresponding parameters will be set to true. The program diagram of threshold comparison module is shown in Figure 8.

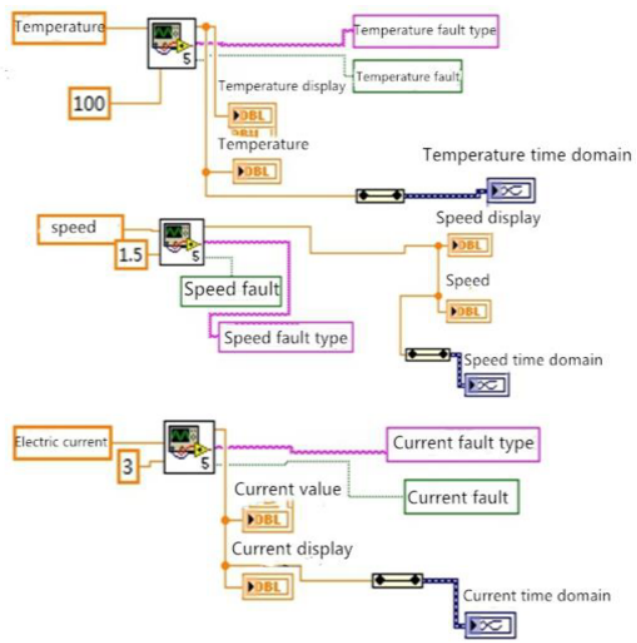

Figure 8. Design of threshold comparison module

\section{Design of Front Panel}

The front panel of LabVIEW main program is mainly divided into three parts: graphics display area, status display area and value display area.

The function of the graphic display area is to display the data including temperature, current, and speed output by receiving end from the single-chip microcomputer and perform time domain display.

The function of the status display area is to compare the data acquired by the sensor with the preset threshold, output the status string of the motor in real time, and set TRUE to the corresponding fault indicator when the motor fails.

The function of the numerical display area is to display the temperature, current and speed data obtained by the current sensor. At the same time, the user can save the three kinds of data. The monitoring interface of the host computer is shown in Figure 9.

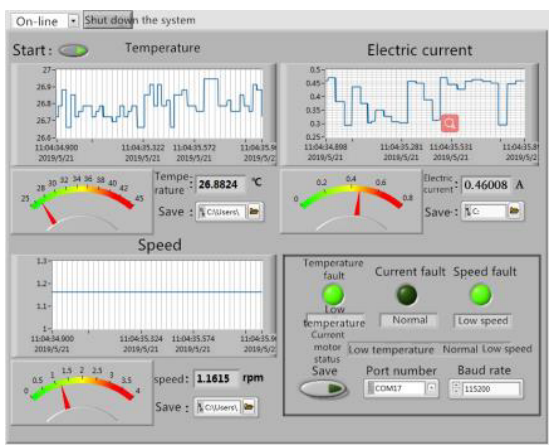

Figure 9. Upper computer monitoring interface 


\section{Conclusion}

The system uses LabVIEW and STM32 as the control core to realize the multi parameter on-line monitoring during the motor operation. The lower computer uses single chip microcomputer, sensor and signal conditioning circuit to complete the acquisition of relevant parameters and signal processing in the process of motor operation. After the data is processed, it is sent to the upper computer through the radio frequency module. Through the upper computer motor monitoring software, data acquisition, reading, display, threshold alarm, data storage and other functions are realized. The system is easy to operate and friendly to interface, and is suitable for on-line monitoring of motor state.

\section{Acknowledgments}

This research is supported by the Natural Science Foundation of China (No:51805168) and Department of education of Jiangxi Province (No:GJJ180301)

\section{Reference}

[1] Shao Y. Design and research of motor monitoring system based on ZigBee technology. Dalian University of technology, 2017

[2] Zheng DY, Zhang PJ. Summary of stator insulation fault diagnosis and online monitoring technology of AC motor. Chinese Journal of electrical engineering, 2019,39 (02): 395-406 + 637

[3] Liang YZ, Gao JL, Deng ZM. Design of linear motor monitoring system based on LabVIEW. Machine tools and hydraulics, 2010,38 (20): 80-82

[4] Zhou LQ, Hu YX, Yao ZQ. Development of real-time signal monitoring system for CNC machine tools based on LabVIEW and compactrio platform. Modular machine tools and automatic processing technology, 2016 (10): 83-86

[5] Liu Y, Wang WX. Design and implementation of three wire PT100 temperature measurement system based on MSP430. Sensors and Microsystems, 2019,38 (03): 75-78

[6] Xu LC, Su CY, Liang YC, Cong PQ, Li FC, Bai LS. Experimental design of DC motor speed monitoring based on LabVIEW. Experimental science and technology, 2013,11 (04): 56-59

[7] Li ZX, Zhou GB, Wang PH, Queen L, Zhang P. Design of intelligent detection system for mechanical parts based on STM32. Modular machine tool and automatic processing technology, 2015 (07): 121-123

[8] Qin ZH. Electrotechnics. Higher education press, 2009. 36-86

[9] Tong SB, Fundamentals of analog electronic technology, higher education press, 2010, 128-169

[10] Cai Y, Sun LB, Jiang WT, Pang L, Zhao PC. Design of real-time online motor monitoring system based on LabVIEW. Instrument technology and sensor, 2017 (10): 70-73 